\title{
INITIAL EXPERIMENTS OF RF GAS PLASMA SOURCE FOR HEAVY ION FUSION*
}

\author{
L. Ahle', R. P. Hall, A.W. Molvik, LLNL, Livermore, CA 94550, USA \\ E. Chacon-Golcher, J. W. Kwan, K, N. Leung, J. Reijonen, LBNL, Berkeley, CA 94720, USA
}

\begin{abstract}
The Source Injector Program for the U.S. Heavy Ion Fusion Virtual National Laboratory is currently exploring the feasibility of using RF gas plasma sources for a HIF driver. This source technology is presently the leading candidate for the multiple aperture concept, in which bright millimeter size beamlets are extracted and accelerated electrostatically up to $1 \mathrm{MeV}$ before the beamlets are allowed to merge and form $1 \mathrm{~A}$ beams. Initial experiments have successfully demonstrated simultaneously high current density, $\sim 100 \mathrm{~mA} / \mathrm{cm}^{2}$, and fast turn on, $\sim 1 \mu \mathrm{s}$. These experiments were also used to explore operating ranges for pressure and RF power. Results from these experiments are presented as well as progress and plans for the next set of experiments for these sources.
\end{abstract}

\section{BEAMLET INJECTOR FOR HIF}

The source injector for a heavy ion fusion driver must deliver $\sim 10^{16}$ ions to the accelerator [1]. Given current limits of sources and transport limits of accelerators, the injector will almost assuredly have multiple beams. Most present conceptual designs have an injector energy of 1.6 $\mathrm{MeV}$ and current per beam of $0.5 \mathrm{~A}$ [2]. Presently, the Heavy Ion Fusion Virtual National Laboratory is pursuing a concept of creating the $0.5 \mathrm{~A}$ beams by initial extracting many, $\sim 100$, small beamlets and accelerating them to 1 $\mathrm{MeV}$ before they are allowed to merge to together [3]. This concept promises to produce brighter, a key figure of merit, higher current beams and a more compact injector. Initial simulations have given encouraging results.

Another advantage of the beamlet architecture is the beam brightness of the merged beam is dominated by the emittance growth of the merging process and, is only a weak function of the ion temperature of the source. This allows the possibility of using sources with higher ion temperatures instead of surface ionization sources. The merged beams in the beamlet injector only achieve high brightness if the current density of the beamlet is high, $\sim 100 \mathrm{~mA} / \mathrm{cm}^{2}$. In fact, current density becomes a more important criterion for the source than ion temperature.

\section{RF PLASMA SOURCE}

For the beamlet concept to be practical, there must be a source that delivers high current density and easily accommodates the geometry of many millimeter size

\footnotetext{
*This work performed under the auspices of US DOE by UC-LLNL under contract W-7405-ENG-48 and by UC-LBNL under contract DEAC03-76SF00098.

†ahle1@1lnl.gov
}

beams. A plasma source is such a source. Specifically, the HIF program has started development of a multicusp RF plasma source for HIF. These sources have been used to produce high current density beams and can form the beamlets by using a single RF bucket with a grid for the extraction plate. In fact, K.N. Leung's group at LBNL demonstrated over $200 \mathrm{~mA} / \mathrm{cm}^{2}$ for $\mathrm{Ar}^{+}$[4].

Another important requirement for the sources in HIF is the ability of a fast turn on. To minimize non-linear effects in the head of the beam, the rise time of the voltage waveform across the first gap must be $\sim 100 \mathrm{~ns}$. Plasma formation times are usually of the order $10 \mu$ s, implying the necessity for a fast high voltage pusler. Even with such a pulser, a fast rise time is not guaranteed. The emission surface in a plasma source does change as the voltage pulse ramps up, so a fast beam current pulse may not arise with a fast high voltage pulse. K.N. Leung's group also demonstrated fast rise time, $\sim 1 \mu \mathrm{s}$, with a RF plasma source, but with only a few microamps of beam current [5]. Thus, HIF source injector program recently conducted experiments aimed at simultaneously demonstrating fast rise time and high current density.

\section{EXPERIMENTAL SETUP}

The experiments were preformed with the same $10 \mathrm{~cm}$ multicusp source used in reference [4]. The plasma chamber has an inner diameter of $10 \mathrm{~cm}$ with $20 \mathrm{SmCo}$ magnet columns. A 1.5-turn, quartz antenna with a $5 \mathrm{~cm}$ diameter coil generates plasma inside the chamber. The power is delivered to the antenna from a RF amplifier via a fifty-ohm transmission line and an impedance matching network. The RF amplifier generates a $2 \mathrm{~ms}$ burst of 13.56 $\mathrm{MHz} \mathrm{RF}$ signal at a rep rate of $10 \mathrm{~Hz}$. The maximum peak RF output power of the amplifier is $5 \mathrm{~kW}$. The gas, Argon, is introduced to the plasma chamber through a needle valve and the absolute pressure is measure by a capacitance manometer. A small tungsten starter filament is used to feed seed electrons and typically drew about $60 \mathrm{~W}$ of power in order to provide an adequate source of electrons.

The extraction system consists of a plasma electrode with an aperture of $3 \mathrm{~mm}$. The gap to the ground electrode is $3 \mathrm{~mm}$, which has a $4 \mathrm{~mm}$ diameter opening for the beam to travel through. A pulser, manufactured by Diversified Electronics, provides the extraction voltage. The system is capable of delivering a $50-\mathrm{kV}, 20-$ microsecond pulse with a peak current of 25 A. A faraday cup with a magnetic filter was used to measure the beam current extracted from the source. The faraday cup picks up transients from the extraction pulser. These transients are subtracted from the data in software by 
recording the signal from the faraday cup with the extraction voltage pulse but without the RF amplifier so that no beam is extracted. Figure 1 shows a raw faraday cup signal with and without the RF amplifier running and the software subtraction of the two. As can be seen from the figure, the transients picked up by the faraday cup is only a small fraction of the signal.

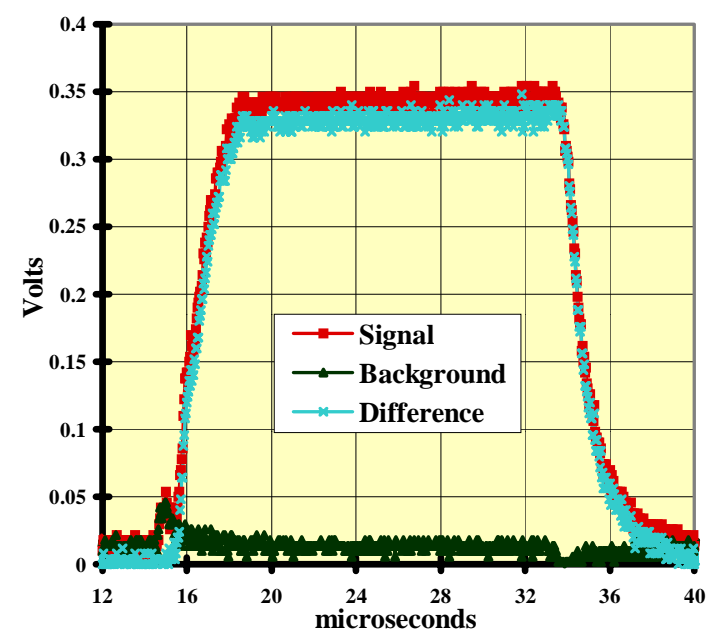

Figure 1: Sample signal from Faraday cup with and without beam extraction and difference between the two.

\section{EXPERIMENTAL RESULTS}

First, it was verified that this source could produce 100 $\mathrm{mA} / \mathrm{cm}^{2}$ of beam current density. The matched condition for the source configuration was determined by varying the extraction voltage and measure the flattop beam current. Figure 2 shows a plot of current density versus extraction voltage. The data shows a typical voltage to the three-halves power behavior for low voltage. In this region, the Child-Langmuir limit for space charge limited ion extraction is lower than the emission limit from the plasma source. At around $15 \mathrm{kV}$, the two limits are near equal. Above, this voltage, the extracted current density plateaus, indicating emission limited beam extraction. For this example, the matched condition was determined to be $14.4 \mathrm{kV}$. This plot clearly shows that $100 \mathrm{~mA} / \mathrm{cm}^{2}$ can be produced by this source.

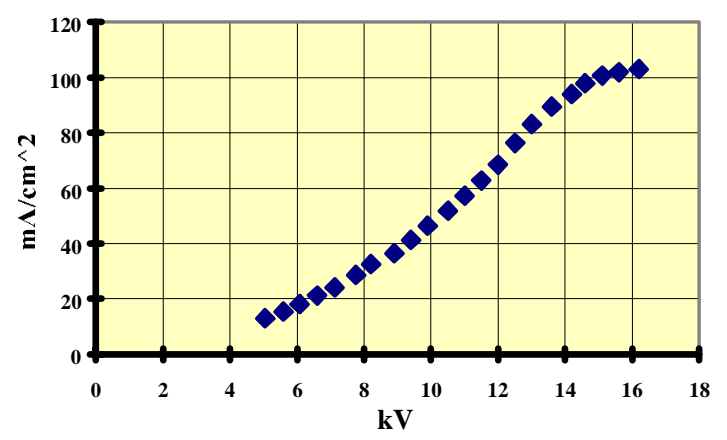

Figure 2: Current density versus extraction voltage.

With the starter filament in place, $2 \mathrm{mT}$ was found to be the minimum pressure for stable operation of the source. Below this pressure, the RF pulse did not always form plasma. Below about $1.0 \mathrm{mT}$, plasma was never ignited. The RF amplifier was set to $3 \mathrm{~kW}$ of peak power for the 2 ms pulse. The extraction pulser was set to fire $500 \mu \mathrm{s}$ after the RF pulse started in order to allow the plasma to stabilize before beam extraction. The extraction voltage was set to $14.4 \mathrm{kV}$.

Figure 3 shows the resulting extraction voltage waveform and extracted current density. The delay between the two is the time of flight of the argon ions to the Faraday cup. The measure current density is 93.3 $\mathrm{mA} / \mathrm{cm}^{2}$. This, however, is not a fundamental limit of the setup, but simply the matched condition for this RF power setting and pressure. Figure 4 is the same plot with the time axis expanded around the front of the pulse. Analysis of the waveforms revealed a $2.2+/-0.1 \mu$ s rise time for $10 \%-90 \%$ of the flattop. The extraction pulse also has a similar rise time, $2.3+/-0.1 \mu \mathrm{s}$, indicating that the rise time is being limit by the pusler and not the plasma source itself.

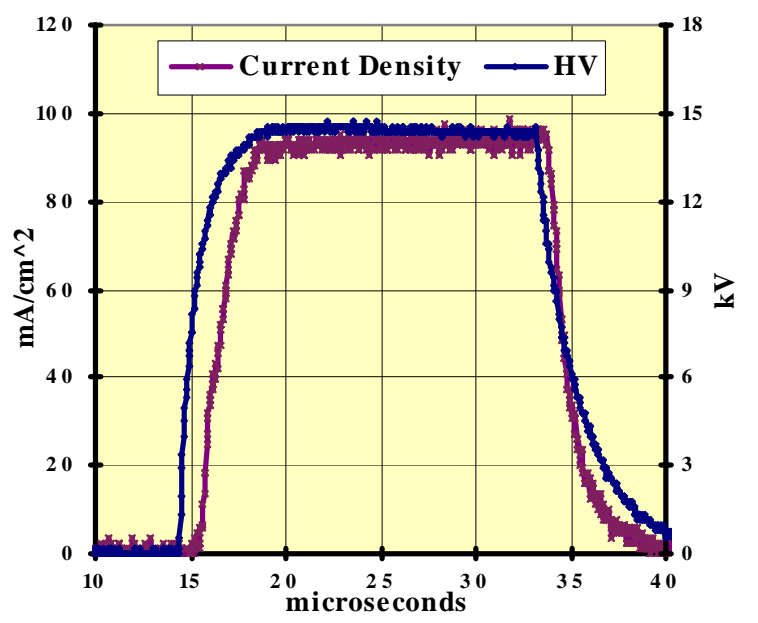

Figure 3: High voltage pulse and extracted current pulse for plasma source with starter filament.

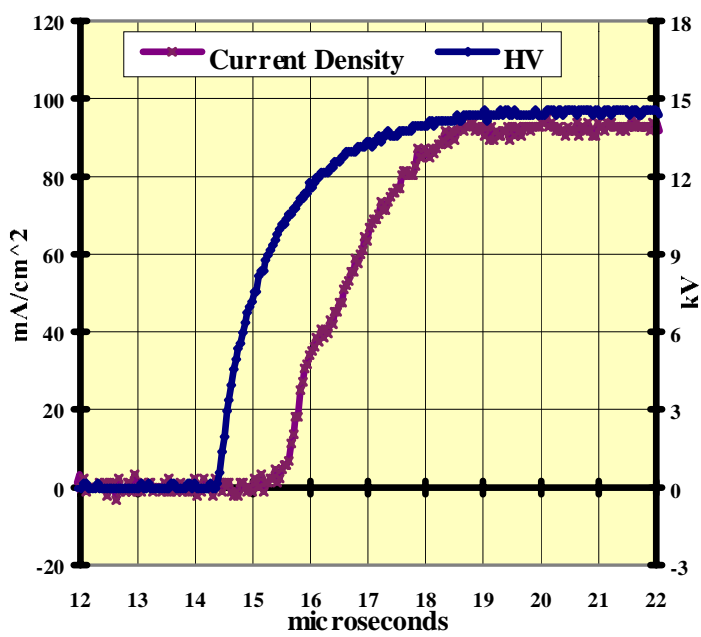

Figure 4: Same as figure 3, only with the time scale expanded around start of pulse.

The rise time of the pulser is dominated by the stray capacitance and inductance of the system. One way to significantly reduce the effect of the load is the remove 
starter filament and the isolation transformer needed to deliver power to it. This was removed from the system and the measurement repeated. Unfortunately, without the starter filament the minimum pressure for stable operation is $18 \mathrm{mT}$. To get a similar current density, the $\mathrm{RF}$ power was reduced to $2 \mathrm{~kW}$. For these settings, a matched condition was achieved at $15.7 \mathrm{kV}$. Figure 5 shows the resulting waveforms. For this configuration the rise time of the extraction pulse is $1.4+/-0.1 \mu \mathrm{s}$. The current pulse again appears to follow the extraction pulse with a rise time of $1.5+/-0.1 \mu \mathrm{s}$. Again, there is no evidence the plasma source itself is limiting the rise time. Unfortunately, further reduction in the rise time of the extraction pulse would require a different pulser or major changes to the test setup.

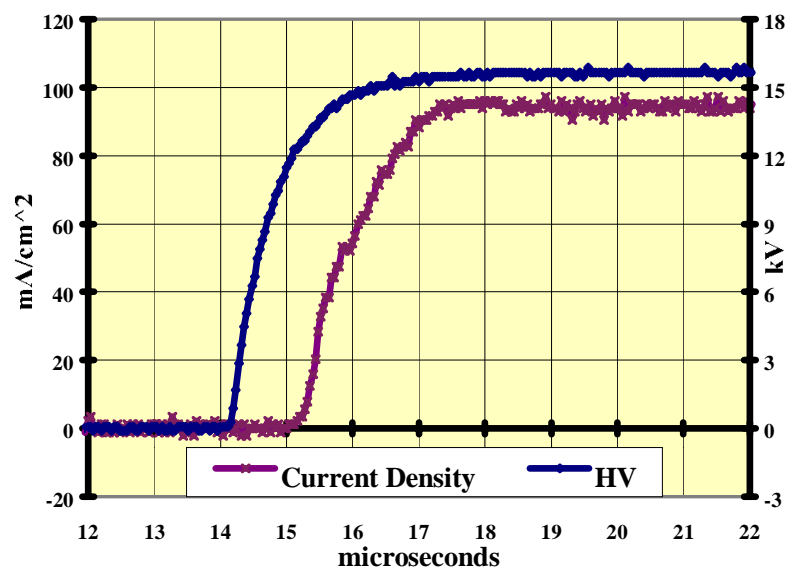

Figure 5: Extraction voltage and current density versus time for plasma source without the starter filament.

\section{DISCUSSION}

These results demonstrate it is possible to achieve simultaneously high current density, $\sim 100 \mathrm{~mA} / \mathrm{cm}^{2}$, and fast rise time, $\sim 1 \mu \mathrm{s}$, with a multicusp RF gas plasma source. The data indicate the rise time is limited by the extraction pulse and not the plasma source itself. Though there may be a plasma source limit below a $1 \mu \mathrm{s}$. This result is achieved by fast switching of the extraction voltage and not the RF power. A starter filament allows for operation at a lower pressure, $2 \mathrm{mT}$ as compared to 18 $\mathrm{mT}$ without. Also only $3 \mathrm{~kW}$ of peak RF power is needed to generate current densities of $100 \mathrm{~mA} / \mathrm{cm}^{2}$. Even though more power would be needed for a source large enough to generate $100,5 \mathrm{~mA}$ beamlets, a duty factor of less than $0.1 \%$ is needed for the RF power, which implies a low average power system is needed.

\section{FUTURE PLANS}

Based on these results, the HIF program has decided to continue development of the plasma source. A larger plasma source capable of delivering 100, $5 \mathrm{~mA}$ beamlets is under construction. The inner diameter of the plasma cavity is $26 \mathrm{~cm}$ and it has 38 magnets surrounding the outside. The antenna is a one and two-thirds turn, quartz antenna with a diameter of $10 \mathrm{~cm}$. Based on the results of the previous source, it is estimated that $\sim 10 \mathrm{~kW}$ of peak $\mathrm{RF}$ power is need to create plasma with a high enough density to extract $100 \mathrm{~mA} / \mathrm{cm}^{2}$. This RF power will be provided by a pulsed RF system, which can be floated at high voltage.

The source will be tested on the source pulser system of the Recirculator [6]. This pulser system is capable of delivering up to $100 \mathrm{kV}$ and rise times of $\sim 300 \mathrm{~ns}$ when driving a $10 \mathrm{nF}$ load. This system may be capable of even faster rise times for loads with less capacitance, as is expected when this source is connected to this platform. This pulser system should allow full scale testing for the first gap of the beamlet architecture. Experiments will be focused on exploring extracted current density and emittance versus RF power and gas pressure. Charge exchange in the extraction gap is an important concern and energy measurements will be performed to determine the magnitude of this effect. Also, charge state purity will be explored with time of flight measurements. A pulsed gas system may also be developed to minimize gas load and thus charge exchange in the extraction gap. Results from the experiments should be available by April of 2002.

\section{REFERENCES}

[1] R. O. Bangerter, Nuovo Cimento 106A (11), 1445 (1993).

[2] J. W. Kwan, et. al., Nuovo Cimento 106A (11), 1541, (1993).

[3] J. W. Kwan, et. al., "Design of a Compact High Current Injector for Heavy Ion Fusion", these proceedings.

[4] J. Reijonen, et. al., Proceedings of 1999 Particle Accelerator Conference, 1943, (1999).

[5] L. T. Perkins, et. al., Review of Scientific Instruments, 69 (2), 1060, (1998).

[6] L. Ahle, et. al., Proceedings of 1999 Particle Accelerator Conference, 3248, (1999). 